\title{
T lymphoblastic lymphoma with BCR-ABL negative chronic myeloid leukaemia: a novel association
}

\author{
Mahwish Faizan ${ }^{1}$, Saadia Anwar ${ }^{1}$, Rahat UI Ain ${ }^{1}$, Huma Zafar ${ }^{1}$, Nazish Saqlain² and Zunaira Rathore ${ }^{3}$ \\ ${ }^{1}$ Department of Paediatric Haematology/Oncology, The Children's Hospital \& Institute of Child Health, Ferozepur Road, Lahore 54400, Pakistan \\ ${ }^{2}$ Department of Haematology, The Children's Hospital \& Institute of Child Health, Ferozepur Road, Lahore 54400, Pakistan \\ ${ }^{3}$ Department of Histopathology, The Children's Hospital \& Institute of Child Health, Ferozepur Road, Lahore 54400, Pakistan
}

\begin{abstract}
Lymphoblastic lymphoma and chronic myeloid leukaemia (CML) are two distinct neoplasms with different pathogenesis and clinical presentation. We hereby share a challenging case of a child presenting with fever, leucocytosis, generalised lymphadenopathy and massive splenomegaly. He was diagnosed as having novel association of concurrent T-lymphoblastic lymphoma diagnosed on cervical lymph node biopsy with BCR-ABL negative $\mathrm{CML}$ on bone marrow aspirate. The study of more such cases is needed for optimal patient management.
\end{abstract}

Keywords: lymphoblastic lymphoma, children, atypical chronic myeloid leukaemia

\section{Introduction}

Acute lymphoblastic leukaemia/lymphoma (LBL) is a clonal hematopoietic stem cell disorder of B or T cell origin. LBL comprise approximately $30 \%$ of childhood non Hodgkin lymphomas. Chronic myeloid leukaemia (CML) constitutes $2 \%-3 \%$ of leukaemia in children. Atypical CML (BCR-ABL negative) is a challenging myeloid malignancy with features of both myeloproliferative and myelodysplastic syndromes. The diagnosis of both myeloid and lymphoid neoplasms in a single patient, whether simultaneous or sequential, is extremely rare, with an overall incidence of less than 1\% [1]. Our reported case has concurrent $\mathrm{T}$ lymphoblastic lymphoma and BCR-ABL negative CML, which have never been reported in the literature before.

\section{Case report}

A 9-year-old child presented to the oncology clinic in September 2020 with a 6 month history of fever, fatigue and gradually increasing bilateral neck swellings. There were no complaints of cough, respiratory distress, bone pains, petechiae or bruises. He was a pale
Correspondence to: Mahwish Faizan Email: mahwishfz@gmail.com

ecancer 2021, 15:1221

https://doi.org/10.3332/ecancer.2021.1221

Published: 22/04/2021

Received: 24/12/2020

Publication costs for this article were supported by ecancer (UK Charity number 1176307).

Copyright: $($ the authors; licensee ecancermedicalscience. This is an Open Access article distributed under the terms of the Creative Commons Attribution License (http:// creativecommons.org/licenses/by/3.0), which permits unrestricted use, distribution, and reproduction in any medium, provided the original work is properly cited. 
looking, active and alert child having weight of $22 \mathrm{~kg}$. A Bacillus Calmatte-Guerin (BCG) vaccine scar was present. He had generalised lymphadenopathy, largest were bilateral cervical lymph nodes 5 by $5 \mathrm{~cm}$, discrete, firm, non-tender with normal overlying skin. His liver was enlarged $4 \mathrm{~cm}$ below right costal margin and spleen was enlarged $15 \mathrm{~cm}$ below left costal margin. The rest of systemic examination was unremarkable. His haemoglobin was $6.9 \mathrm{gm} / \mathrm{dL}$, White blood cells (WBC) $168.4 \times 10^{3} / \mathrm{L}$, neutrophils $36 \%$, lymphocytes $02 \%$, eosinophil's $07 \%$, monocytes $02 \%$, promyelocytes $02 \%$, myelocytes $26 \%$, metamyelocytes $16 \%$, basophils $07 \%$, blasts $02 \%$ and mean corpuscular volume (MCV) 80.1 $\mathrm{fL}$, mean corpuscular hemoglobin (MCH) $26.4 \mathrm{pg}$, Peripheral blood film shows macrocytosis, anisocytosis and poikilocytosis. Nucleated red blood cells 03/100 WBC. No hemoparasites were identified. Platelets 72,000/L. serum uric acid $7.2 \mathrm{md} / \mathrm{dL}$, potassium $4 \mathrm{mmol} / \mathrm{L}$, latcate dehydrogenase (LDH) 780. Echocardiography, hepatitis B \& C screening, calcium, phosphate, liver and renal function tests were normal. CT neck, chest, abdomen showed bilateral cervical (largest measures $6 \mathrm{~cm}$ by $5 \mathrm{~cm}$ ), axillary, supraclavicular, mediastinal and abdominal lymphadenopathy, hepatosplenomegaly (L $15 \mathrm{~cm}, S 17.2 \mathrm{~cm}$ ) and splenic parenchymal defects suggesting lymphoproliferative disorder with incidental note of left pelvi ureteral junction (PUJ) obstruction. Flow cytometry on bone marrow aspirate was inconclusive showing less than $5 \%$ blasts. Bone marrow aspirate showed hypercellular fragments showing active trilineage haematopoiesis. Plasma cells constitute $3 \%$ of nucleated marrow cells. No hemoparasites identified. Blasts cell constitute $05 \%$ of nucleated marrow cells. BM differential was myelocytes $40 \%$, metamyelocytes $02 \%$, erythroids $05 \%$, neutrophils $45 \%$ and blasts cells $08 \%$. Bone marrow trephine biopsy was adequate length with normal bony trabeculae, reduced fat spaces and $95 \%$ cellularity (Figure 1). Erythropoiesis was suppressed with hyperplastic myelopoiesis and megakaryopoiesis seen. Peripheral blood film and bone marrow findings were suggestive of chronic myeloproliferative neoplasm (MPN) most likely chronic myelogenous leukaemia in chronic Phase.

Excisional biopsy of cervical lymph node was pan T (CD3) positive, pan B (CD20) negative, Tdt positive, CD117 negative, cyclin D-1 negative and $\mathrm{KI} 67 \mathrm{High}(>80 \%)$ consistent with T cell lymphoblastic lymphoma/leukaemia (Figure 2). BCR-ABL translocation was not detected on fluorescence in situ hybridization (FISH) and real-time polymerase chain reaction (RT-PCR). Differential diagnosis considered included CML with Lymphoma, lymphadenopathy as an extramedullary manifestation of CML or myeloid sarcoma. Considering the rarity of association of T lymphoblastic lymphoma/leukaemia with BCR-ABL negative CML in paediatric age group, both the cervical lymph node biopsy and bone marrow aspiration biopsy were sent for external review, after discussion in tumour board meeting. Both reports corresponded to and confirmed the primary diagnosis of T lymphoblastic lymphoma with BCR-ABL negative CML which is unheard of in the paediatric population. The family was appropriately counselled. The child was started on tumour lysis prophylaxis, Hydration and pneumocystis jerovecci prophylaxis. The literature was searched to make optimal treatment plan for this child. There was a paucity of data to make exact recommendation of the treatment. The family was counselled about bone marrow transplant when his lymphoblastic lymphoma was in remission, to which they did not consent. The child was started on UKALL 2011 Regimen C protocol. Four Drug Induction completed on 1st January 2021. Currently, the child is on Capizzi Interim Maintenance chemotherapy and doing well. Post induction workup showed haemoglobin was $10.8 \mathrm{gm} / \mathrm{dL}$, WBC $24.78 * 10^{3} / \mathrm{L}$, neutrophils $50 \%$, lymphocytes $06 \%$, eosinophil's $04 \%$, monocytes $05 \%$, promyelocytes $02 \%$, myelocytes $24 \%$, metamyelocytes $9 \%$, basophils $01 \%$ and blasts $01 \%$. CT scan neck chest and abdomen show few subcentimeter size lymph nodes in neck, more on left side with subcentimetric axillary and supraclavicular, mesenteric lymph nodes. Right kidney is normal. Left kidney shows PUJ obstruction. Liver enlarged $14 \mathrm{~cm}$ and spleen measures $14.2 \mathrm{~cm}$ markedly enlarged. Repeat bone marrow (induction day 8 \& day 29) still favour MPN likely CML in chronic phase. Currently, clinically, he is doing well with no palpable lymphadenopathy; however, splenomegaly persists $4 \mathrm{~cm}$ (from $15 \mathrm{~cm}$ baseline) below left costal margin, though markedly regressed. His lymphoblastic Lymphoma seems to be in remission while atypical chronic myeloid leukaemia $(\mathrm{aCML})$ in chronic phase persists.

\section{Discussion}

Lymphoma is the third most common childhood malignancy accounting for $10 \%-12 \%$ of childhood cancers. Non-Hodgkin lymphoma (NHL) account for $7 \%$ of cancers in children $<20$ years of age [2]. T-cell lymphoblastic lymphoma/leukaemia (T-LBL) is the most common paediatric T cell Lymphoma. They develop in lymphoid tissues such as lymph nodes and spleen or outside of lymphoid tissues like gastrointestinal tract, liver, nasal cavity, skin etc. CML is rare in children and constitutes $2 \%$ of all leukaemias in children younger than 15 years and $9 \%$ of all leukaemias in adolescents between 15 and 19 years [3]. About $90 \%-95 \%$ of children with CML have a genetic alteration called the Philadelphia chromosome. aCML is a rare myelodysplastic syndrome/MPN in children. 


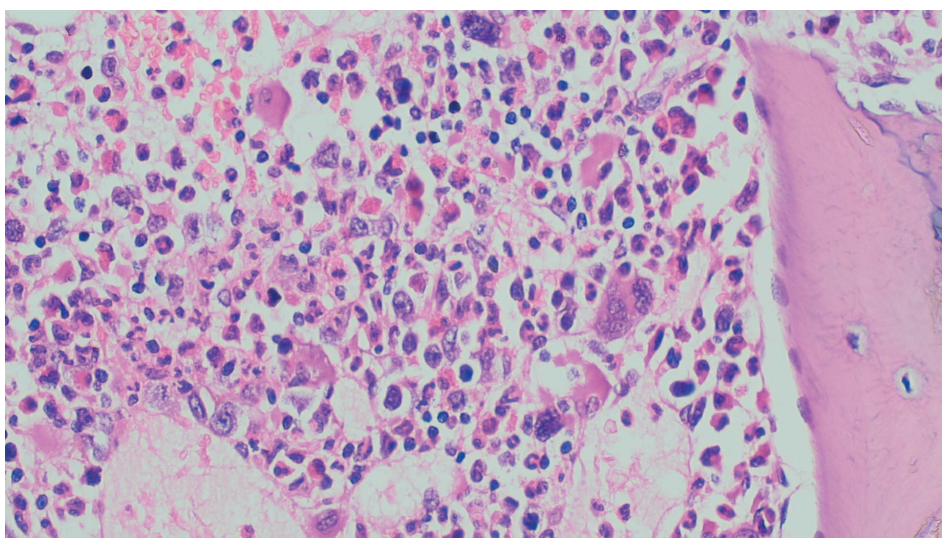

Figure 1. Bone marrow trephine biopsy.

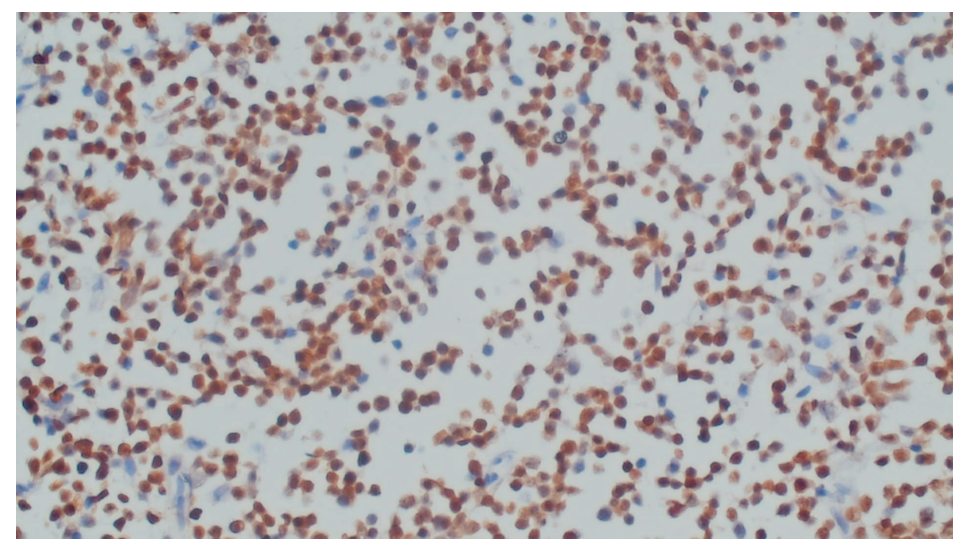

Figure 2. Cervical lymph node biopsy (terminal deoxynucleotidyl transferase/Tdt positive).

Morphology-based criteria are currently used to diagnose it. The main feature of aCML is the presence of neutrophilic leucocytosis and marked dysgranulopoiesis. Further diagnostic criteria include $\mathrm{WBC} \geq 13 \times 10^{\%} / \mathrm{L}$ with $\geq 10 \%$ of immature granulocytes, and $\leq 20 \%$ blasts in the blood and the bone marrow $[4,5]$. To rule out other MPNs, BCR-ABL1, platelet-derived growth factor receptor alpha (PDGFRA), platelet-derived growth factor receptor beta (PDGFRB), and fibroblast growth factor receptor (FGFR) rearrangements should be excluded in all cases [6].

There has been a case report of secondary malignancies in CML [7]. Fu et al [8] reported simultaneous diagnosis of T-LBL and BCR-ABL positive CML in two adult patients who were treated with imatinib and HYPER-CVAD chemotherapy. Simultaneous acute myeloid leukaemia (AML) and lymphoma has been reported in the literature and is more frequent than simultaneous CML and lymphoma [9]. Melikian et al [10] reported 20 adult patients with synchronous and metachronous tumors.There are also case reports of coexistence of T cell Lymphoblastic lymphoma co existing with atypical myeloproliferative disorder associated with t(8:13) (p21;q14) [11].

Our index patient fulfilled the criteria of both precursor T-NHL based on immunohistochemistry and typical morphology on lymph node biopsy and BCR-ABL negative CML. This association has not been previously described in the literature either in adults or children. Due to the rarity of this co existence, little is known about pathogenesis and optimal treatment plan. 
Precursor T-NHL is an aggressive malignancy but the development of intensified T-ALL-focused protocols has resulted in significant improvements in outcome in children [12]. No standard of care is currently available for the management of co existent aCML. Drugs called tyrosine kinase inhibitors are the first line of treatment for BCR-ABL positive CML. However, the only curative treatment for atypical CML remains haematopoietic stem cell transplant (HSCT) [13]. Participation in clinical trials should be considered in all cases. Different treatment options like hypomethylating agents (azacytidine or decitabine) [14], Hydroxy urea, Interferon alpha, AML like therapy or targeted therapies (Janus kinase gene (JAK) inhibitor ruxolitinib, the SRC (proto-oncogene tyrosine-protein) kinase inhibitor dasatinib, and the MEK inhibitor trametinib) have been used with variable results in patients not suitable for bone marrow transplant $[15,16]$. The prognosis of the concurrent bi-lineage malignancies is generally poorly understood and chemotherapy is necessary. More research is required to optimize management for such children.

\section{Conclusion}

T lymphoblastic lymphoma with atypical CML is an unusual association. The T LBL component regressed completely in the index child after treatment with T ALL based therapy. More data is required to optimize management for such children.

\section{Acknowledgments}

Haematology Lab team, Agha Khan university Hospital, Karachi, Pakistan for secondary review \& Paediatric Oncology Eastern and Mediterranean Group (POEM) for MDT discussion.

\section{Conflicts of interest}

No conflicts of interest.

\section{Funding}

No funding was received for this case report for any purpose.

\section{References}

1. Hauck G, Jonigk D, and Kreipe H, et al (2013) Simultaneous and sequential concurrent myeloproliferative and lymphoproliferative neoplasms Acta Haematol 129 187-196 https://doi.org/10.1159/000342484

2. (2008) Cancer in children (ages 0-14 and ages 0-19) SEER Cancer Statistics Review, 1975-2006 eds MJ Horner, LAG Ries, and M Krapcho (Bethesda: National Cancer Institute)

3. Ries LAG, Smith M, and Gurney JG, et al (1999) Cancer incidence and survival among children and adolescents: United States SEER program 1975-1995 SEER Program vol 99 (Bethesda: National Cancer Institute) pp 46-49

4. Vardiman JW, Thiele J, and Arber DA, et al (2009) The 2008 revision of the World Health Organization (WHO) classification of myeloid neoplasms and acute leukemia: rationale and important changes Blood 114 937-951 https://doi.org/10.1182/blood-2009-03-209262 PMID: 19357394 
5. Gotlib J, Maxson JE, and George TI, et al (2013) The new genetics of chronic neutrophilic leukemia and atypical CML: implications for diagnosis and treatment Blood 122 1707-1711 https://doi.org/10.1182/blood-2013-05-500959 PMID: 23896413 PMCID: 3765056

6. Arber DA, Orazi A, and Hasserjian R, et al (2016) The 2016 revision to the World Health Organization classification of myeloid neoplasms and acute leukemia Blood 127 2391-2405 https://doi.org/10.1182/blood-2016-03-643544 PMID: 27069254

7. Miranda MB, Lauseker M, and Kraus M-P, et al (2016) Secondary malignancies in chronic myeloid leukemia patients after imatinib-based treatment: long-term observation in CML Study IV Leukemia 30 1255-1262 https://doi.org/10.1038/leu.2016.20 PMID: 26859076 PMCID: 4895174

8. Fu X, Shang Y, and Zhang L, et al (2018) Analyses and treatment of simultaneous bi-lineage malignancies of myeloid leukemia and lymphoma: two case reports and a literature review Oncol Lett 16(5) 6624-6632 https://doi.org/10.3892/ol.2018.9447 PMID: 30405801 PMCID: $\underline{6202486}$

9. Ichinohasama R, Miura I, and Takahashi N, et al (2000) Ph-negative non-Hodgkin's lymphoma occurring in chronic phase of Ph-positive chronic myelogenous leukemia is defined as a genetically different neoplasm from extramedullary localized blast crisis: report of two cases and review of the literature Leukemia 14 169-182 https://doi.org/10.1038/sj.leu.2401606 PMID: 10637493

10. Melikian AL Kolosheinnova TI, and Goriacheva SR, et al (2014) Synchronous and metachronous myeloid and lymphoid tumours Ter Arkh $8637-44$

11. Somers GR, Slaters H, and Rockman S, et al (1997) Coexistent T-cell lymphoblastic lymphoma and an atypical myeloproliferative disorder associated with t(8;13)(p21;q14) Pediatr Pathol Lab Med 17 141-158 https://doi.org/10.1080/15513819709168354 PMID: 9050068

12. Teachey DT and O'Connor D (2020) How I treat newly diagnosed T-cell acute lymphoblastic leukemia and T-cell lymphoblastic lymphoma in children Blood 135 159-166 https://doi.org/10.1182/blood.2019001557 PMCID: 6966932

13. Onida F, de Wreede LC, and van Biezen A, et al (2017) Allogeneic stem cell transplantation in patients with atypical chronic myeloid leukaemia: a retrospective study from the Chronic Malignancies Working Party of the European Society for Blood and Marrow Transplantation Br J Haematol 177 759-765 https://doi.org/10.1111/bjh.14619 PMID: 28369779

14. Jiang H, Wu Z, and Ren L, et al (2016) Decitabine for the treatment of atypical chronic myeloid leukemia: a report of two cases Oncol Lett 11 689-692 https://doi.org/10.3892/ol.2015.3977 PMID: 26870268 PMCID: 4726938

15. Freedman JL, Desai AV, and Bailey LC, et al (2016) Atypical chronic myeloid leukemia in two paediatric patients Pediatr Blood Cancer 63 156-159 https://doi.org/10.1002/pbc.25694

16. Crisa E, Nicolosi M, and Ferri V, et al (2020) Atypical chronic myeloid leukemia: where are we now? Int J Mol Sci 21(18) 6862 https://doi. org/10.3390/ijms21186862 PMCID: 7555965 